\title{
Extensa lesión necrotizante de la línea media inducida por uso de cocaína
}

\section{Extensive cocaine-induced necrotizing midline destructive lesion}

\author{
Francisco García-Huidobro N. ${ }^{1}$, Andrés Rosenbaum F. ${ }^{1}$, Pablo Cabello E. ${ }^{1}$, \\ Sebastián Bravo-Grau ${ }^{2}$, Sofía Waissbluth A. ${ }^{1}$
}

Departamento de Otorrinolaringología, Red de Salud UC Christus, Pontificia Universidad Católica de Chile. Santiago, Chile.

${ }^{2}$ Departamento de Radiología, Red de Salud UC Christus, Pontificia Universidad Católica de Chile. Santiago, Chile.

Los autores declaran no tener conflictos de interés.

Recibido el 29 de abril de 2020. Aceptado el 4 de Julio de 2020 .

Correspondencia: Sofía Waissbluth A

Departamento de Otorrinolaringología Red de Salud UC Christus, Pontificia Universidad Católica de Chile

Marcoleta 352

Santiago, Chile. Email: sofia.waissbluth@gmail.

\section{Resumen}

El diagnóstico diferencial de las lesiones destructivas de la línea media es amplio y complejo debido a su presentación clínica poco específica, pudiendo abarcar desde cuadros infecciosos, neoplásicos y patologías autoinmunes. Una entidad a considerar son las lesiones destructivas de la línea media inducidas por cocaína (CIMDL), la que se caracteriza por una extensa destrucción de estructuras a nivel medio-facial. Se presenta el caso de un paciente con antecedentes de consumo de cocaína con una gran lesión destructiva endonasal asociada a una perforación palatal, fiebre, cervicalgia y meningismo. Se realizó un estudio nasofibroscópico y exámenes de laboratorio donde destaca un leve aumento de parámetros inflamatorios y serología positiva para anticuerpos anticitoplasma de neutrófilo (ANCA). Los exámenes imagenológicos destacan una trombosis de carótida interna derecha, osteítis del clivus y paquimeningitis retroclival. Se decide ingresar a pabellón para realizar un aseo y tomar biopsias. Al descartar los diagnósticos diferenciales de CIMDL, el paciente inició terapia antibiótica y antifúngica endovenosa evolucionando en forma satisfactoria. La CIMDL es una condición infrecuente cuyo principal diagnóstico diferencial es la granulomatosis con poliangeitis. Dada similitud en las presentaciones clínicas y la gran diferencia en el manejo de ambas patologías la necesidad de un diagnóstico certero es fundamental.

Palabras clave: lesión necrotizante, fístula oronasal, cocaína, vasculitis, lesión de la línea media.

\begin{abstract}
Differential diagnosis of destructive midline injuries is complex due to its nonspecific clinical presentation, and the broad variety of conditions that share the same signs and symptoms, such as infections, neoplasms and autoimmune diseases. One of the etiologies that should be considered is cocaine-induced destructive midline lesions (CIMDL), characterized by extensive destruction of midface structures. The following case describes a patient with history of cocaine abuse with a large destructive endonasal lesion associated with a palatal perforation, fever, cervicalgia and meningism. Clinical assessment included a nasofibroscopy and laboratory tests. Results revealed a slight increase in inflammatory parameters and positive antineutrophil cytoplasmic antibody serology. Additionally, imaging revealed a thrombosis of the right internal carotid, clivus osteitis, and retroclival pachymeningitis. Surgical debridement was performed, and biopsies of the nasal cavity were taken. Once the other possible diagnoses were ruled out, the patient was started on intravenous antibiotic and antifungal therapies. The patient showed a positive response to treatment and was successfully discharged after two months. CIMDL is a rare condition whose main differential diagnosis is granulomatosis with polyangiitis. Given the similarity in clinical presentations and the important differences in the management of both pathologies, the need for an accurate diagnosis is essential.
\end{abstract}

Keywords: necrotizing lesion, oronasal fistula, cocaine, vasculitis, midline lesion. 


\section{Introducción}

Las lesiones destructivas de la línea media a nivel nasal son consideradas entidades sindromáticas de diagnóstico complejo ${ }^{1}$. Esto debido a la extensa variedad de etiologías y a que la presentación clínica es poco específica, ya que muchos signos y síntomas descritos son comunes para otros cuadros como infecciones, neoplasias y patologías autoinmunes ${ }^{1,2}$. Uno de los diagnósticos a considerar son las lesiones destructivas de la línea media inducidas por consumo de cocaína (CIMDL, sigla en inglés: cocaine induced midline destructive lesions $)^{1}$. Esta complicación secundaria al abuso de esta sustancia se caracteriza por la presencia de lesiones ulcerativas necrotizantes, gran cantidad de costras y perforaciones septales ${ }^{2,3}$. Si bien la CIMDL ha sido ampliamente descrita en múltiples publicaciones, los mecanismos por los cuales se producen estas lesiones aún son desconocidos $^{3,5}$. El consenso actual es que su patogenia podría ser multifactorial, incluyendo fenómenos isquémicos, traumáticos, apoptóticos, súper-infecciones y/o cambios autoinmunes asociados a vasculitis ${ }^{6,9}$. La asociación con estos últimos ha sido ampliamente reportada, siendo la CIMDL una de las principales formas de presentación de pseudovasculitis inducida por cocaína en conjunto con la vasculitis inducida por levamisol, antihelmíntico presente como aditivo a esta droga en un $70 \%$ de los ca$\operatorname{sos}^{4,5,8}$. Además, se ha observado en un porcentaje no menor de casos de CIMDL la presencia de anticuerpos anticitoplasma de neutrófilos
(ANCA), los cuales son muy sugerentes de vasculitis idiopáticas de pequeño vaso como la granulomatosis con poliangeitis (GPA), previamente conocida como granulomatosis de Wegener ${ }^{8}$. Este antecedente dificulta aún más el diagnóstico etiológico de esta patología, ya que ambos cuadros clínicos son similares y generalmente el abuso de esta droga no es referido en forma espontánea por el paciente. A continuación, presentamos el caso clínico de un paciente con una extensa lesión destructiva de línea media inducida por cocaína.

\section{Caso Clínico}

Paciente de sexo masculino de 47 años con antecedentes de rinitis atrófica severa por supuesto consumo crónico de cocaína, suspendido hace un mes, se presenta en el Servicio de Urgencia derivado por su médico tratante por historia de un mes de evolución de compromiso del estado general y cefalea, al que se agrega cervicalgia progresiva con rigidez cervical y sensación febril de tres días de evolución. Al examen físico destaca subfebril, taquicárdico y con signos de meningismo, por lo cual se hospitaliza. Por los antecedentes mencionados, se solicita evaluación por el equipo de otorrinolaringología realizando una nasofibrosocopía en la que destaca una extensa lesión destructiva que compromete las fosas nasales y paladar blando (Figura 1). Además, a la otoscopía se observa tubos $\mathrm{T}$ bilaterales permeables.

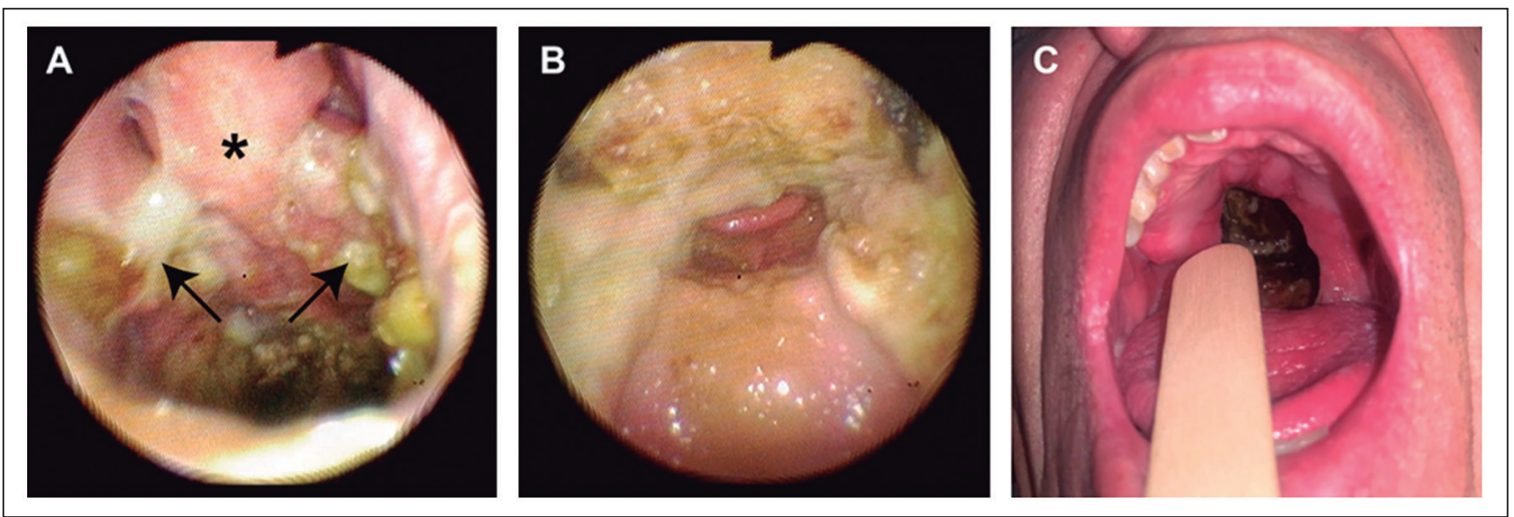

Figura 1. A: Vista endoscópica panorámica de fosas nasales observando abundantes costras y necrosis, remanente de tabique (asterisco) con ausencia de cornetes inferiores y destrucción parcial de cornetes medios (flechas). B: Vista endoscópica desde rinofaringe observando abundantes costras y necrosis con destrucción completa de paladar blando. C: A través de cavidad oral, se observa destrucción de paladar blando. 
En los exámenes de laboratorio (Tabla 1), destaca anemia, leucocitosis con un predominio de neutrófilos y trombocitosis. La prueba de proteína $C$ reactiva estaba levemente elevada. Exámenes inmunológicos revelan positividad para c-ANCA (citoplasmático). Por la observación de meningismo, se realiza una punción lumbar la cual arroja un cultivo negativo y examen citoquímico sin alteraciones. La tomografía computarizada (TC) de cerebro y cuello mostró perforación septal, alteración de densidad de la grasa entre el clivus y odontoides con presencia de gas y pérdida de la definición de la cortical en pared anterior del clivus (Figuras 2A y 2B).

La resonancia magnética (RM) de cerebro mostró oclusión de arteria carótida interna derecha, con repermeabilización en segmento supraclinoídeo, osteítis con componente necrótico del clivus y paquimeningitis retroclival (Figuras 2C y 2D). Con estos hallazgos, se decide con conjunto con el equipo tratante, tomar biopsias de la mucosa nasal y palatina para estudio de lesiones destructivas de la línea media, lo cual se realiza en pabellón. Se toman múltiples muestras de cornete medio derecho, etmoides y remanente de paladar duro en cuyo análisis destaca desarrollo de Staphylococcus aureus, Staphylococcus epidermidis y Candida glabrata (cultivo micológico). Las tinciones con calcoflúor y Kinyoun resultaron negativas, al igual que el cultivo de Nocardia. En la
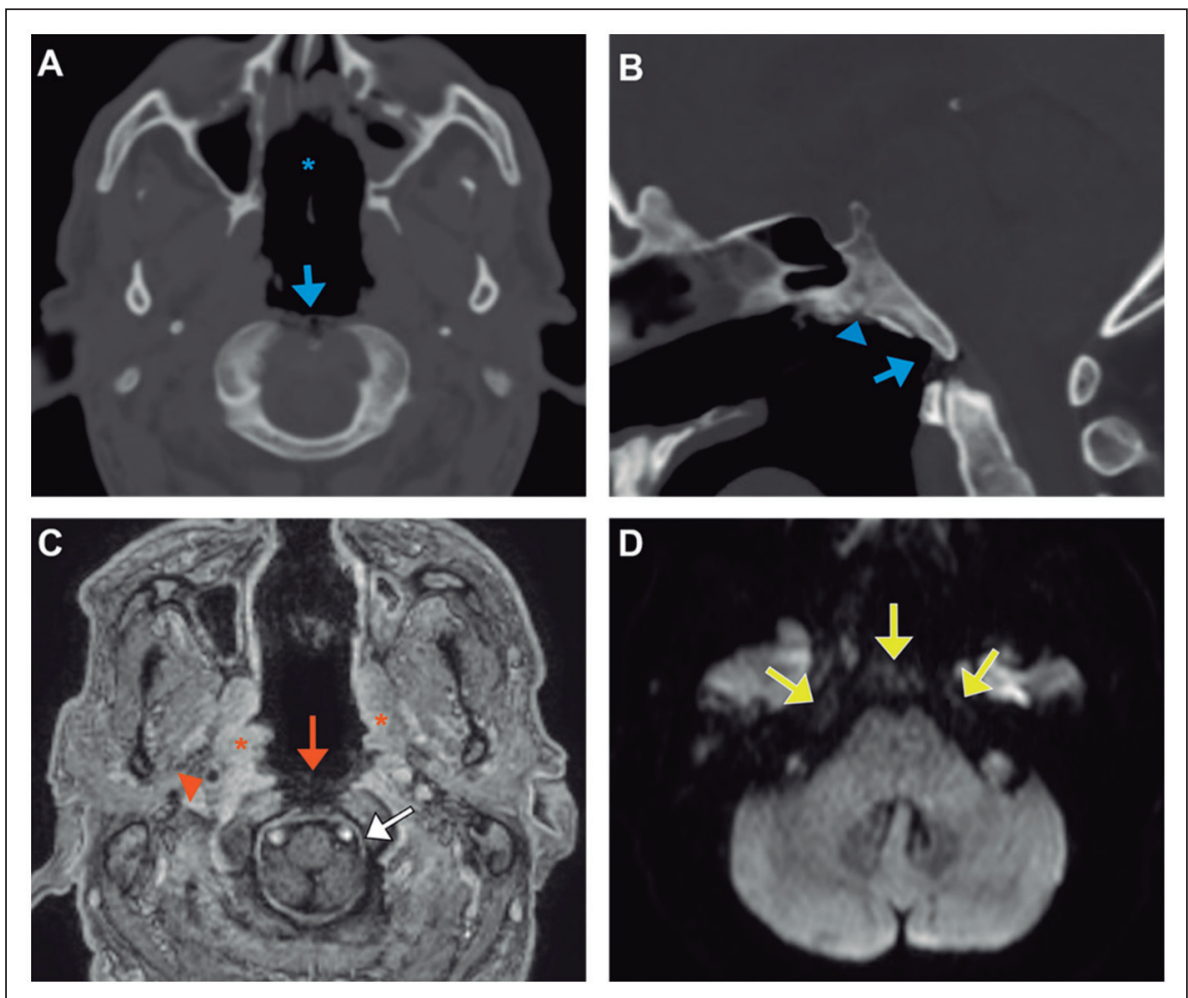

Figura 2. TC de cerebro, corte axial (A) y sagital (B), que muestra extensa perforación del tabique nasal (asterisco azul) así como marcado adelgazamiento de la mucosa rinofaríngea y orofaríngea posterior, asociado a múltiples pequeñas burbujas de gas en el aspecto superior de la articulación atlanto-odontoidea (flechas azules). Alteración del patrón óseo trabecular del clivus con pérdida de definición de la cortical anterior (cabeza de flecha azul). RM de cerebro, T1 con contraste (reformateo axial) (C): ausencia de refuerzo mucoso rino y orofaríngeo posterior, traduce necrosis mucosa (flecha naranja). Defecto de llenado en segmento cervical de la arteria carótida interna derecha secundario a trombosis (cabeza de flecha naranja). Engrosamiento y mayor refuerzo del resto de la mucosa visible, cambios inflamatorios e hiperémicos por consumo de drogas simpaticomiméticas (asteriscos). Refuerzo paquimeníngeo liso retroclival y en el nivel de la cisterna magna (flecha blanca), secundario a compromiso inflamatorio dural. (D) DWI (B 1000), restricción a la difusión de la médula ósea del clivus y apex petroso, en conjunto con los hallazgos de TC, están en el contexto de osteitis (flechas amarillas). 




citometría de flujo no se observaron elementos de clonalidad sugerentes de compromiso por un síndrome linfoproliferativo. También se enviaron muestras para estudio anatomopatológico que mostraron fragmentos de mucosa orofaríngea con ulceración e inflamación crónica con focos supurados. Se evidenciaron elementos levaduriformes y pseudohifas, pero sin angioinvasión. No se observaron colonias bacterianas. En los fragmentos de mucosa nasal, se observó lámina propia con abundante infiltrado linfocitario y neutrofílico. No se observaron granulomas.

Se concluyen los diagnósticos de osteítis de base de cráneo y unión craneocervical con extensión atlantoaxial, paquimeningitis retroclival, trombosis séptica de la arteria carótida derecha, seno cavernoso y petroso inferior 


\section{Tabla 2. Diagnóstico diferencial de lesiones destructivas de la línea media}

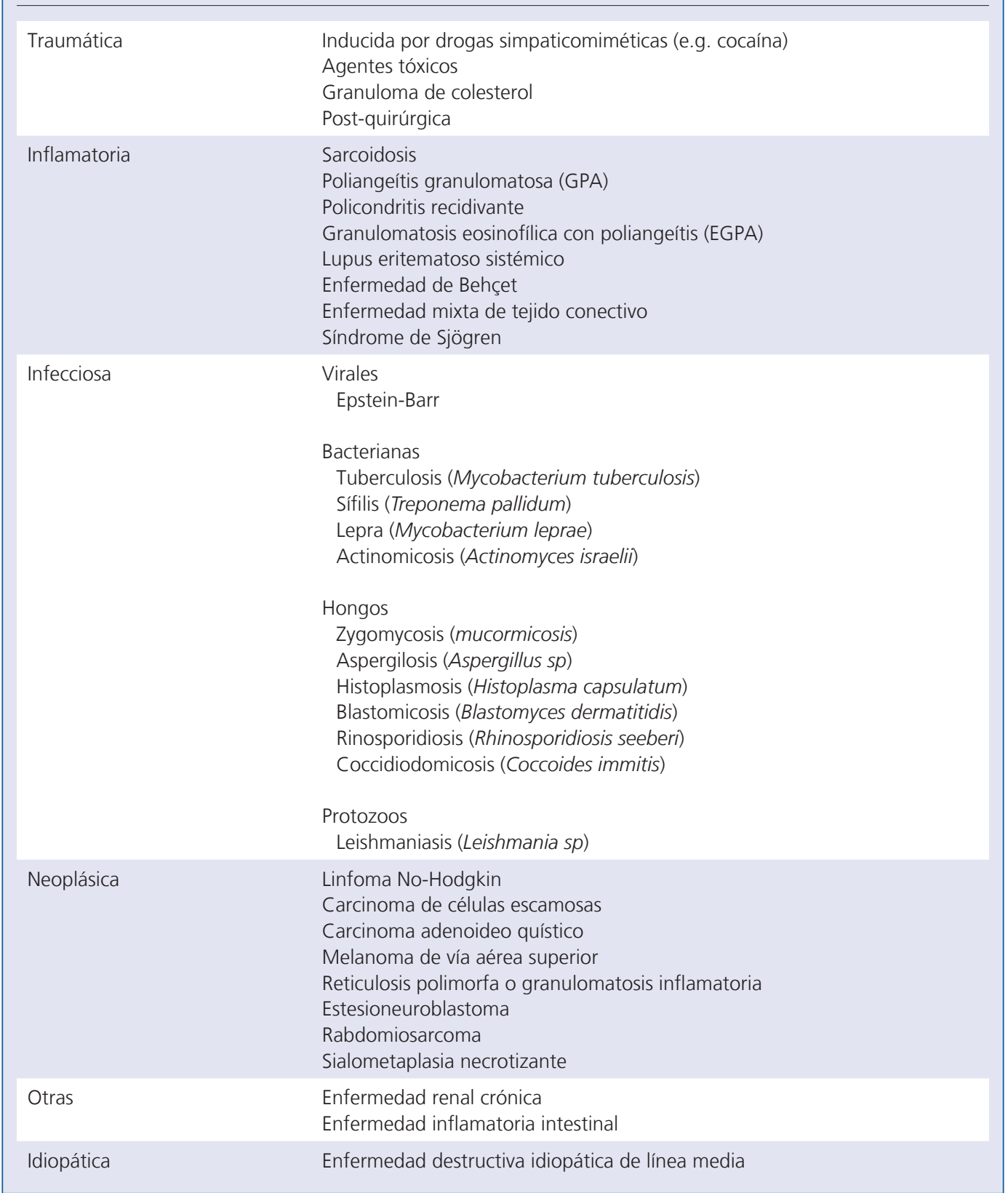

derecho. El paciente se mantuvo hospitalizado por un mes con anidulafungina y enoxaparina. Se indicó cefazolina, cloxacilina y metronidazol durante 21 días, luego cambiándose a meropenem. Evoluciona favorablemente y es dado de alta, con acenocumarol según esquema, amoxicilina-ácido clavulánico y fluconazol. Al mes posterior al alta, el paciente se encontraba estable en términos de síntomas rinosinusales y lesión de línea media. Actualmente, el paciente se encuentra en control por medicina interna, neurología y cirugía maxilofacial, y en seguimiento mensual por nuestra especialidad. 


\section{Discusión}

La CIMDL es una complicación poco frecuente que se presenta en un $4,8 \%$ de los consumidores activos de cocaína, caracterizándose por una extensa destrucción de estructuras osteocartilaginosas a nivel medio-facial ${ }^{2,3,10}$. Esta presentación no es exclusiva de esta patología y puede ser producida por una gran variedad de condiciones las cuales deben ser excluidas al momento de realizar un diagnóstico definitivo (Tabla 2) ) $^{3,11,12}$. Uno de los principales diagnósticos diferenciales es la GPA, dado que ambas comparten características que las hacen difíciles de diferenciar ${ }^{5,13}$. Por esta razón, una historia clínica detallada, un examen físico acucioso y exámenes de laboratorio e imagenológicos cobran gran importancia ${ }^{5,14}$.

Desde el punto de vista clínico, en ausencia del antecedente de consumo, Barrera y cols. plantean que la distribución de las lesiones podría orientar el diagnóstico, ya que la CIMDL presenta una destrucción local más extensa en comparación con las lesiones de $\mathrm{GPA}^{4}$. Esto ha sido reafirmado por Trimarchi y cols., los cuales indican en base a estudios con TC y RM que, además de la extensión, existe un patrón de erosión más excéntrico en los pacientes con CIMDL $^{2}$. Tanto la extensión como el patrón de erosión fueron observados en nuestro caso (Figura 2) lo que se tradujo finalmente en un compromiso paquimeningeo retroclival secundario, complicación que también ha sido descrita por otros autores ${ }^{7,15}$. Además, otro hallazgo clínico sugerente de CIMDL de acuerdo con lo descrito por Trimarchi y cols., es la perforación palatal, ya que una revisión de la literatura indica que este hallazgo se ha descrito en 73 pacientes con CIMDL, y solo hay 5 reportes en pacientes con GPA ${ }^{10}$.

En relación al uso de exámenes de laboratorio se recomienda solicitar metabolitos de cocaína en orina, sangre y/o pelo si se sospecha CIMDL, ya que los pacientes habitualmente no entregan ese antecedente ${ }^{2}$. En nuestro caso no fue necesario ya que el paciente indicó el consumo en forma espontánea. Por otro lado, también se solicitan títulos de ANCA como herramienta diagnóstica en la evaluación de GPA, sin embargo, se ha descrito que hasta un $84 \%$ de los pacientes con CIMDL pueden presentar valores positivos, lo que dificulta aún más la diferenciación entre estas patologías 5 . Este fenómeno aún no tiene una etiología clara, sin embargo, se ha planteado que puede ser producida por la presencia de infecciones por Staphylococcus aureus (asociadas a recidiva de GPA) o por vasculitis inducida por levamisol. Este fármaco se utiliza para tratar infecciones parasitarias, sin embargo, también es encontrado como agregado en la cocaína ya que potencia y prolonga la acción de las catecolaminas inhibiendo el efecto de la monoamino oxidasa y la catecol-o-metiltransferasa ${ }^{8}$. Esto permite prolongar el efecto euforizante $y$, además, le agrega peso sin alterar su pureza ${ }^{5,8}$. Se ha visto que en pacientes con vasculitis inducida por esta droga pueden encontrarse títulos elevados de p-ANCA (86\%-100\%) y c-ANCA (50\%) en pacientes con CIMDL ${ }^{5,8}$. A pesar de lo anteriormente expuesto, se ha visto que los pacientes con CIMDL presentan con mayor frecuencia títulos positivos de anticuerpos anti-mieloperoxidasa (anti-MPO/p-ANCA) y además, que la presencia de resultados positivos de anticuerpos anti-proteinasa 3 (antiPR3/c-ANCA), los cuales son característicos de GPA, pueden presentarse en la mitad de los casos por reacción cruzada ante anticuerpos de elastasa neutrofílica humana (HNE ANCA $)^{8}$. Esto se produce debido a que tanto PR3 como HNE pertenecen a la misma familia de proteasas séricas ${ }^{8}$. Si bien se ha visto que el HNE ANCA se encuentra presente en casi la totalidad de los pacientes con CIMDL, y que no hay presencia de este en GPA, este recurso no se encuentra ampliamente disponible $e^{8,11,13}$. Este no fue solicitado como parte del estudio de nuestro paciente por no estar disponible en nuestro centro, sin embargo, se encontraron títulos positivos de anti-PR3 c-ANCA.

\section{Conclusión}

La CIMDL representa una complicación poco frecuente del consumo de cocaína la cual tiene una presentación clínica cuyos síntomas pueden superponerse e incluso confundirse con algunas patologías autoinmunes como la GPA. Teniendo en consideración lo difícil que es diferenciar ambas entidades diagnósticas, la realización de un estudio acabado que incluya una evaluación clínica exhaustiva asociada 
a exámenes de laboratorio e imagenológicos cobra gran importancia.

\section{Bibliografía}

1. Parker NP, Pearlman AN, Conley DB, Kern RC, Chandra RK. The dilemma of midline destructive lesions: a case series and diagnostic review. Am J Otolaryngol - Head Neck Med Surg. 2010;31(2):104109. doi:10.1016/j.amjoto.2008.11.010.

2. Trimarchi M, Bertazzoni G, Bussi M. Cocaine induced midline destructive lesions. Rhinology. 2014;52(2):104-111. doi:10.4193/Rhino13.112.

3. Trimarchi M, Bussi M, Sinico RA, Meroni P, Specks U. Cocaine-induced midline destructive lesions - An autoimmune disease? Autoimmun Rev. 2013;12(4):496-500. doi:10.1016/j. autrev.2012.08.009.

4. Barrera OM, Rivera VM de la, Vela UJ, Barrera MR, Mönckeberg FG. Lesiones destructivas de la línea media facial secundarias al consumo de cocaína. Caso clínico. Rev Med Chile 2018;146(9):1070-1073. doi:10.4067/s0034-98872018000901070.

5. Mirzaei A, Zabihiyeganeh M, Haqiqi A. Differentiation of cocaine-induced midline destructive lesions from ANCA-associated vasculitis. Iran J Otorhinolaryngol. 2018;30(5):309-313. doi:10.22038/ijorl.2018.25210.1817.

6. Trimarchi M, Miluzio A, Nicolai P, Morassi ML, Bussi M, Marchisio PC. Massive apoptosis erodes nasal mucosa of cocaine abusers. Am J Rhinol. 2006;20(2):160-164. doi:10.1177/194589240602000207.

7. Molteni M, Saibene AM, Luciano K, MacCari A. Snorting the clivus away: An extreme case of cocaineinduced midline destructive lesion. BMJ Case Rep.
2016;2016. doi:10.1136/bcr-2016-216393.

8. Berman M, Paran D, Elkayam O. Cocaineinduced Vasculitis. Rambam Maimonides Med J. 2016;7(4):e0036. doi:10.5041/rmmj.10263.

9. Smith JC, Kacker A, Anand VK. Midline nasal and hard palate destruction in cocaine abusers and cocaine's role in rhinologic practice. Ear, Nose Throat J. 2002;81(3):172-177. doi:10.1177/014556130208100313.

10. Trimarchi M, Bondi S, Della Torre E, Terreni MR, Bussi M. La perforazione del palato differenzia le lesioni destruenti della linea mediana indotte da cocaina dalla granulomatosi con poliangioite. Acta Otorhinolaryngol Ital. 2017;37(4):281-285. doi:10.14639/0392-100X-1586.

11. Laudien M. Orphan diseases of the nose and paranasal sinuses: Pathogenesis - clinic - therapy. GMS Curr Top Otorhinolaryngol Head Neck Surg. 2015;14:Doc04. doi:10.3205/cto000119.

12. Orden A, Yöchler A, Ejarque A, et al. Lesión destructiva de la línea media. Granulomatosis de Wegener. Rev Argentina la Soc Argentina Reumatol. 2011;22(3):2011:42-54.

13. Subesinghe S, van Leuven S, Yalakki L, Sangle S, D'Cruz D. Cocaine and ANCA associated vasculitislike syndromes-A case series. Autoimmun Rev. 2018;17(1):73-77. doi:10.1016/j.autrev.2017.11.011.

14. Trimarchi M, Gregorini G, Facchetti F, et al. Cocaine-induced midline destructive lesions: Clinical, radiographic, histopathologic, and serologic features and their differentiation from Wegener granulomatosis. Medicine (Baltimore). 2001;80(6):391-404. doi:10.1097/00005792200111000-00005.

15. Gatt K, Vella SM, Fenech M, Mallia Azzopardi C. Infective complications of midline destruction in a cocaine user. BMJ Case Rep. 2019;12(7):1-3. doi:10.1136/bcr-2019-231743. 